MATEC Web of Conferences 16, 07004 (2014)

DOI: $10.1051 /$ matecconf/ 20141607004

(C) Owned by the authors, published by EDP Sciences, 2014

\title{
Quasi-coordinates based dynamics control design for constrained systems
}

\author{
Elżbieta M. Jarzębowska \\ Warsaw University of Technology, Institute of Aeronautics and Applied Mechanics, \\ 00-665 Warsaw, Nowowiejska 24 St., Poland, e-mail:elajarz@meil.pw.edu.pl
}

\begin{abstract}
The paper presents model-based dynamics control design for constrained systems which exploits dynamics modeling in quasi-coordinates. These non-inertial coordinates are useful in motion description of constrained systems as well as in a controller design, since they offer many advantages in both areas. Specifically, dynamics model formulation results in a reduced-state form of the motion equations. The selection of quasi-coordinates is arbitrary so they may satisfy the constraint equations and be control inputs directly. The paper presents an approach to control oriented modeling and a controller design based on the generalized Boltzmann-Hamel equations where the generalization refers to constraint kinds which may be put upon systems, i.e. constraints may be material or artificial like control constraints. The control design framework applies to fully actuated and underactuated systems and it is computationally efficient. Examples of controller designs and their comparisons to a traditional, Lagrange model- based framework are presented.
\end{abstract}

\section{Introduction}

The paper presents model-based control design for constrained systems which uses dynamics modeling in quasi-coordinates. The constrained systems may be subjected to holonomic, nonholonomic or programmed constraints as well as be fully actuated or underactauted. Such systems are a large class of systems of a practical interest and they are usually approached by the Lagrange method with generalized coordinates or its modifications to obtain their motion equations. The Lagrange based dynamics are then used to generate dynamic control models for these systems. This traditional, almost routine, approach to dynamics modeling results in dynamics that lacks some properties significant from the point of view of further control design. Basically, Lagrange based dynamics can be applied to systems with constraints of first order and the number of unknowns that result from Lagrange's equations increases to include the multipliers. In order to obtain a dynamic control model, Lagrange's based dynamics require the elimination of the constraint reaction forces (Lagrange multipliers). Finally, solutions obtained from the Lagrange based models require numerical stabilization due to differentiation of constraint equations, which may complicate on-line simulations and control. Only a few works report using a quasi-coordinate approach to modeling systems, see e.g. $[1,2]$.

From the perspective of mechanics and derivation of equations of motion constrained systems may belong to the same class, e.g. be subjected to first order nonholonomic constraints. From the perspective of nonlinear control theory, they may differ and may not be approached by the same control strategies and algorithms. Their control properties depend upon the way they are designed and propelled. Then, from the nonlinear control theory perspective a system design, way of its propulsion, control goals, other motion or work-space constraints may determine the way of the control-oriented modeling.

The dynamics modeling in quasi-coordinates presented herein, which is incorporated in the modelbased control design for constrained systems eliminates many disadvantages related to Lagrange's based dynamics modeling and a subsequent control design.

Motivations for the development of constrained and control dynamics in quasi-coordinates comes from the author experience in area of modeling and control of constrained systems. Firstly, the constraint kinds that have to be dealt with in control setting are different than the ones considered in analytical modeling. This has led to the formulation of the unified constraint formulation and the generalized programmed motion equations $[3,4]$. Secondly, a dynamics control model that is passed to a control engineer to design and apply to it an appropriate controller, may be made a control oriented, i.e. may facilitate this controller design. The two motivations are not separate from each other. They both can be appropriately treated at the modeling step of a control design project using the latest modeling tools and the modeling process may serve an effective control design.

In the paper we present the theoretic model-based control oriented modeling framework. It yields equations of motion for constrained systems in quasi-coordinates. It is based on the generalized Boltzmann-Hamel equations [3]. This dynamics framework yields equations of motion of a constrained system in a reduced-state form, from which the dynamic control model directly follows. The framework applies to fully actuated and underactuated systems, it is computationally efficient, and may facilitate a subsequent controller design. Based on the framework, a tracking control strategy dedicated to track predefined 
motions referred to as programmed is designed [5]. It can be redesigned to constrained and control dynamics developed in quasi-coordinates.

The paper contribution is then three folded. Firstly, the model-based control oriented framework for the generation of dynamics for constrained systems formulated in quasi-coordinates, where additionally relations between generalized velocities and quasivelocities may be nonlinear, is presented. Secondly, the dynamics formulation in quasi-coordinates is unified in the sense that it is suitable for systems constrained by any order bilateral constraints. Thirdly, based on this formulation a tracking controller for the system motion along a prescribed programmed motion may be designed.

Examples that illustrate the theory demonstrate the effectiveness of the model-based control oriented modeling framework in quasi-coordinates.

\section{An extended constraint concept - material and non-material constraints imposed upon system motions}

A control design process consists of three main steps, which are a dynamic model building, a control algorithm design, and a controller implementation. Starting from the model building, constraints imposed on a system should be specified first, and inspected if they are holonomic or nonholonomic. We do not address dynamics modeling and control design of holonomic systems, since these are considered solved problems, at least theoretically [6].

Based on the examples of constraints reported in mechanics and control, we start a control-oriented modeling from a revisited constraint concept. An extended understanding of constraints is suitable for both dynamics modeling and control applications. The constraints can be classified as follows [4,5]:

1. Material nonholonomic constraints (NC) - they come from an assumption about rolling vehicle wheels without slipping. They are first order and they are typical for wheeled mobile vehicles, multi-finger hands working on surfaces. Their common form is as

$$
\varphi_{\beta}\left(t, q_{1}, \ldots, q_{n}, \dot{q}_{1}, \ldots, \dot{q}_{n}\right)=0 \quad \beta=1, \ldots, b, \quad b<n
$$

Functions $\varphi_{\beta}$, are defined on a $(2 n+1)$-dimensional manifold and have continuous derivatives. Often, the kinematic constraints are linear in velocities, i.e.

$$
\sum_{\sigma=1}^{n} b_{\beta \sigma}\left(t, q_{1}, \ldots, q_{n}\right) \dot{q}_{\sigma}+b_{\beta o}\left(t, q_{1}, \ldots, q_{n}\right)=0,
$$

Constraints (1) or (2) restrict accelerations but not positions. They are referred to as first order constraints. In classical mechanics setting they are known as material constraints $[7,8]$.

2. Conservation laws - they come from the angular momentum conservation for free floating space manipulators or for a sportsman in an exercise flying phase. Their equation form is the same as (1) [9]. Notice, that in mechanics they are not referred to as constraints. They show up in a control setting.

3. Tasks (programmed constraints) - they can be formulated for any physical system, e.g. a robot or a manipulator and they can specify a task, work to do or a limitation in a system motion, e.g. a limitation in velocity or acceleration. Also, it may specify a trajectory to follow but then it is a holonomic constraint. Many task formulations are reported in [10-13]. However, none of the tasks is formulated in algebraic or differential constraint equation forms at a system modeling level; such equations are formulated later at a level of a controller design and then a specific controller modification for each task is needed the most often. The earliest formulation of programmed constraints (PC) known to the author was given by Appell in [14]. He described them as constraints "that can be realized not through a direct contact". Similar ideas were introduced by Mieszczerski at the beginning of the 20-th century. Beghin developed a concept of servo-constraints [15]. These new "constraint sources" motivated to specify constraints by the formulations like

$$
\varphi_{\beta}\left(t, q_{1}, \ldots, q_{n}, \dot{q}_{1}, \ldots, \dot{q}_{n}\right)=0, \quad \beta=1, \ldots, k, \quad k<n
$$

The history of evolution of the PC (3) confirms both their usefulness in formulations of requirements for dynamical systems performance and leads to a formulation of a "unified constraint formulation", which is

$$
B_{\beta}\left(t, q, \dot{q}, \ldots, q^{(p)}\right)=0, \quad \beta=1, \ldots, k, k<n
$$

where $p$ is a constraint order and $B_{\beta}$ is a $k$-dimensional vector. Equations (4) can be nonlinear in $q^{(p)}$. Differentiation of (4) with respect to time, until the highest derivative of a coordinate is linear, results in constraint equations linear with respect to this highest coordinate derivative. We assume that " $p "$ stands for the highest order derivative of a coordinate which appears linearly in a constraint equation. For simplicity we assume that they are linear in all $p$-th order derivatives of $q$ 's and we rewrite (4) as

$$
B\left(t, q, \dot{q}, \ldots, q^{(p-1)}\right) q^{(p)}+s\left(t, q, \dot{q}, \ldots, q^{(p-1)}\right)=0,
$$

where $B$ is a $(k \times n)$-dimensional full rank matrix, $n>k$, and $s$ is a $(k \times 1)$-vector. The constraint (5) is referred to as a unified constraint formulation [4].

4. Design or control constraints - they can be put upon manipulators and robots with underactuated degrees of freedom [16]. They have the form (5) with $p=2$.

5. Other design, control or operation constraints on robots, manipulators and other vehicles or robotic systems, which can be presented as (5):

in navigation of wheeled mobile robots, to avoid the wheel slippage and mechanical shock during motion, dynamic constraints such as acceleration limits have to be imposed [10,11],

in path planning problems, for car-like robots, to secure motion smoothness two additional constraints are added: on a trajectory curvature and its time derivative so additional constraints of the second and third order are imposed [11],

- in manipulator trajectory tracking, jerk must be limited for reducing manipulator wear and improving tracking accuracy [17],

- in vehicle dynamics constraints are added when different maneuvers are to be performed [18],

- bounded lateral acceleration - e.g. path tracking experiments depend on the precision of the odometry. If 
the lateral acceleration of the vehicle is too large, the wheels can lose close contact to the ground and the odometry data is no longer meaningful [19].

The constraint classification in classical mechanics and a variety of requirements on system's motions reported in the literature can be summarized as follows:

- Many problems are formulated as synthesis problems and motion requirements may be viewed as nonmaterial constraints imposed on a system before it is designed and put into operation.

- Constraints that specify motion requirements may be of orders higher than one or two.

- Non-material constraints may arise in modeling and analysis of electro and biomechanical systems.

- No unified approach to the specification of nonmaterial constraints or any other unified constraint has been formulated in classical mechanics.

These conclusions lead to an idea of an extended constraint concept [4]. It is formulated in two definitions: Definition 1: A programmed constraint is any requirement put on a physical system motion specified by an equation (5).

Definition 2: A programmed motion is a system motion that satisfies a programmed constraint (5).

A system can be subjected to both material and programmed constraints. Programmed constraints do not have to be satisfied during all motion of a system.

\section{3 control oriented constrained dynamics formulation in quasi-coordinates}

Nonholonomic systems (NS) are a large class of systems. From the perspective of mechanics and derivation of equations of motion for them, many of them belong to the same class of systems subjected to first order nonholonomic constraints. They may be approached by Lagrange's equations with multipliers and these equations are used to generate dynamic control models for them most often [8,20,21]. From the perspective of nonlinear control theory, NS differ and may not be approached by the same control strategies and algorithms. Some of them may be controlled at the kinematic level and the other at the dynamic level only. Their control properties depend upon the way they are designed and propelled. Usually, they are divided into two control groups, which are treated separately, the group of fully actuated and the group of underactuated NS $[7,8,16]$.

The constrained dynamics which we formulate below can be directly use as a control dynamics, and serves both fully actuated and underactuated systems constrained by the constraints (5) [4].

Let us start from recalling the concepts of quasicoordinates and quasi-velocities. They were introduced to derive the Boltzmann-Hamel equations of motion. Relations between the generalized velocities and quasivelocities were assumed linear and non-integrable, i.e.

$$
\omega_{r}=\omega_{r}\left(t, q_{\sigma}, \dot{q}_{\sigma}\right), \quad \sigma, r=1, \ldots, n, \quad(6)
$$

With respect to the extended constraint concept (5), our first step is to let (6) be nonlinear [3]. Inverse transformations for (6) can be computed as

$$
\dot{q}_{\lambda}=\dot{q}_{\lambda}\left(t, q_{\sigma}, \omega_{r}\right) . \quad \lambda=1, \ldots, n
$$

Quasi-coordinates can be introduced as

$$
d \pi_{r}=\sum_{\sigma=1}^{n} \frac{\partial \omega_{r}}{\partial \dot{q}_{\sigma}} d q_{\sigma}, \quad r=1, \ldots, n
$$

and (8) are non-integrable. Based on (6)-(8), $q$ 's and $\omega$ 's are related as

$$
d q_{\lambda}=\sum_{\mu=1}^{n} \frac{\partial \dot{q}_{\lambda}}{\partial \omega_{\mu}} d \pi_{\mu} . \quad \lambda=1, \ldots, n
$$

The principal form of the dynamics motion equation [4] has the form

$$
\frac{d}{d t} \sum_{\sigma=1}^{n} p_{\sigma} \delta q_{\sigma}=\delta T+\sum_{\sigma=1}^{n} Q_{\sigma} \delta q_{\sigma}+\sum_{\sigma=1}^{n} p_{\sigma}\left[\left(\delta q_{\sigma}\right)-\delta \dot{q}_{\sigma}\right] \delta q_{\sigma}
$$

Transforming its left and right hand side terms using the relations between $\delta \pi_{r}$ and $\delta q_{\lambda}$ we obtain

$$
\begin{aligned}
& \frac{d}{d t} \sum_{\mu=1}^{n} \tilde{p}_{\mu} \delta \pi_{\mu}=\delta \tilde{T}+\sum_{\mu=1}^{n} \tilde{Q}_{\mu} \delta \pi_{\mu}+ \\
& \sum_{r=1}^{n} \tilde{p}_{r}\left[\left(\delta \pi_{r}\right)-\delta \omega_{r}\right]-\sum_{r=1}^{n} \tilde{p}_{r} \sum_{\mu=1}^{n} W_{\mu}^{r} \delta \pi_{\mu}
\end{aligned}
$$

which is the principal form of the equation of motion in quasi-coordinates for nonlinear $\omega_{r}=\omega_{r}\left(t, q_{\sigma}, \dot{q}_{\sigma}\right) . W_{\mu}^{r}$ are generalized Boltzmann symbols. Quantities $\tilde{p}_{\mu}, \tilde{T}$, $\widetilde{Q}_{\mu}$ are all written in quasi-coordinates.

The generalized form of the Boltzmann-Hamel equations can be derived based on (11). It has the form

$$
\sum_{\mu=1}^{n}\left[\frac{d}{d t}\left(\frac{\partial \tilde{T}}{\partial \omega_{\mu}}\right)-\frac{\partial \tilde{T}}{\partial \pi_{\mu}}+\sum_{r=1}^{n} \frac{\partial \tilde{T}}{\partial \omega_{r}} W_{\mu}^{r}-\tilde{Q}_{\mu}\right] \delta \pi_{\mu}=0 .
$$

For a system subjected to material or programmed NC of the form

$$
\omega_{\beta}=\omega_{\beta}\left(t, q_{\sigma}, \dot{q}_{\sigma}\right)=0 \quad \beta=1, \ldots, b
$$

relations

$$
\delta \pi_{\beta}=\sum_{\sigma=1}^{n} \frac{\partial \omega_{\beta}}{\partial \dot{q}_{\sigma}} \delta q_{\sigma}=0, \quad \beta=1, \ldots, b
$$

hold for all $\omega_{\beta}$. A system has $(n-b)$ degrees of freedom and variations $\delta \pi_{b+1}, \ldots, \delta \pi_{n}$ are independent. Then, $(n-b)$ equations of motion, based on (12), have the form

$$
\frac{d}{d t}\left(\frac{\partial \tilde{T}}{\partial \omega_{\mu}}\right)-\frac{\partial \tilde{T}}{\partial \pi_{\mu}}+\sum_{r=1}^{n} \frac{\partial \tilde{T}}{\partial \omega_{r}} W_{\mu}^{r}=\tilde{Q}_{\mu} \quad \mu=b+1, \ldots, n
$$

to which $n$ kinematic relations

$$
\dot{q}_{\lambda}=\dot{q}_{\lambda}\left(t, q_{\sigma}, \omega_{r}\right), \quad \sigma, \lambda=1, \ldots, n, r=b+1, \ldots, n
$$

have to be added.

Equations (15) are the generalized Boltzmann -Hamel equations for a NS. Notice that $b$ of $\omega$ 's are satisfied based on the constraint equations (16). The rest of quasivelocities are selected arbitrarily by a designer. Equations (15) and (16) can be presented as

$$
\begin{gathered}
M(q) \dot{\omega}+C(q, \omega)+D(q)=\tilde{Q}, \\
B(q, \omega)=0 .
\end{gathered}
$$

A system dynamics control model follows directly from (17) since they are free from the constraint reaction forces

$$
\begin{gathered}
M(q) \dot{\omega}+C(q, \omega)+D(q)=\tilde{Q}+\tilde{\tau}, \\
B(q, \omega)=0 .
\end{gathered}
$$


Equations (15) have to be extended to be applicable to systems subjected to NC of high order given by (5). To enable this, the following lemma can be formulated [4].

Lemma: For a function $\widetilde{F}$ of the form

$$
\tilde{F}=\tilde{F}\left(t, q_{\sigma}, \omega_{r}\right), \quad \sigma, r=1, \ldots, n
$$

where $q_{\sigma}$ and $\omega_{r}$ are related by $\omega_{r}=\omega_{r}\left(t, q_{\sigma}, \dot{q}_{\sigma}\right)$, the following identity holds

$$
\frac{d}{d t}\left(\frac{\partial \tilde{F}}{\partial \omega_{\sigma}}\right)=\frac{1}{p}\left(\frac{\partial \tilde{F}^{(p)}}{\partial \omega_{\sigma}^{(p-1)}}-\frac{\partial \tilde{F}}{\partial \pi_{\sigma}}\right) \cdot p=1,2,3, . .
$$

The proof is by mathematical induction [4]. If we replace $\tilde{F}$ by $\tilde{T}=\tilde{T}\left(t, q_{\sigma}, \omega_{\sigma}\right)$ in (19) and insert it into the generalized Boltzmann-Hamel equations (12), we get

$$
\begin{array}{r}
\frac{1}{p}\left[\frac{\partial \tilde{T}^{(p)}}{\partial \omega_{\mu}^{(p-1)}}-(p+1) \frac{\partial \tilde{T}}{\partial \pi_{\mu}}\right]+\sum_{r=1}^{n} \frac{\partial \tilde{T}}{\partial \omega_{r}} W_{\mu}^{r}=\tilde{Q}_{\mu} \cdot \quad \\
\mu=1, \ldots, n, p=1,2,3
\end{array}
$$

Equations (21) are the extended form of the BoltzmannHamel equations. Now, modify them for systems with $\mathrm{NC}$ of high order

$$
\tilde{G}_{\beta}\left(t, q_{\sigma}, \omega_{r}, \dot{\omega}_{r}, \ldots, \omega_{r}^{(p-1)}\right)=0 .
$$

$$
\beta=1, \ldots, b, \quad \sigma, r=1, \ldots, n, b<n
$$

Based on the generalized definition of the virtual displacement

$$
\delta G_{\beta}=\sum_{\sigma=1}^{n} \frac{\partial G_{\beta}}{\partial q_{\sigma}^{(p)}} \delta q_{\sigma}=0,
$$

where $G_{\beta}=G_{\beta}\left(t, q_{\sigma}, \dot{q}_{\sigma}, \ldots, q_{\sigma}^{(p)}\right)$ are constraints of p-th order specified in $q$ 's, we obtain that

$$
\delta \tilde{G}_{\beta}=\sum_{r=1}^{n} \frac{\partial \tilde{G}_{\beta}}{\partial \omega_{r}^{(p-1)}} \delta \pi_{r}=0 .
$$

In the constraint equation (22) we may partition the vector $\omega^{(p-1)}$ as $\omega^{(p-1)}=\left(\begin{array}{ll}\omega_{\beta}^{(p-1)} & \omega_{\mu}^{(p-1)}\end{array}\right)$ with

$$
\omega_{\beta}^{(p-1)}=\Omega_{\beta}^{(p-1)}\left(t, q_{\sigma}, \omega_{\sigma}, \omega_{\sigma}, \ldots, \omega_{\mu}^{(p-1)}\right) .
$$

By differentiating (25) with respect to time we obtain

$$
\omega_{\beta}^{p}=\Omega_{\beta}^{p}\left(t, q_{\sigma}, \omega_{\sigma}, \omega_{\sigma}, \ldots, \omega_{\mu}^{(p-1)}, \omega_{\mu}^{p}\right) .
$$

Now, using the lemma result we rewrite (12) in the form

$$
\begin{aligned}
& \sum_{\beta=1}^{b}\left\{\frac{1}{p}\left[\frac{\partial \tilde{T}^{(p)}}{\partial \omega_{\beta}^{(p-1)}}-(p+1) \frac{\partial \tilde{T}}{\partial \pi_{\beta}}\right]+\sum_{r=1}^{n} \frac{\partial \tilde{T}}{\partial \omega_{r}} W_{\beta}^{r}-\tilde{Q}_{\beta}\right\} \delta \pi_{\beta}+ \\
& +\sum_{\mu=b+1}^{n}\left\{\frac{1}{p}\left[\frac{\partial \tilde{T}^{(p)}}{\partial \omega_{\mu}^{(p-1)}}-(p+1) \frac{\partial \tilde{T}}{\partial \pi_{\mu}}\right]+\sum_{r=1}^{n} \frac{\partial \tilde{T}}{\partial \omega_{r}} W_{\mu}^{r}-\tilde{Q}_{\mu}\right\} \delta \pi_{\mu}=0 .
\end{aligned}
$$

Based on (24) we have that

$$
\delta \pi_{\beta}=\sum_{\mu=b+1}^{n} \frac{\partial \Omega_{\beta}^{(p-1)}}{\partial \omega_{\mu}^{(p-1)}} \delta \pi_{\mu} \quad \beta=1, \ldots, b
$$

and then (27) takes the form for $\mu=b+1, \ldots, n$

$$
\begin{aligned}
& \frac{1}{p}\left[\frac{\partial \tilde{T}^{(p)}}{\partial \omega_{\mu}^{(p-1)}}-(p+1) \frac{\partial \tilde{T}}{\partial \pi_{\mu}}\right]+\sum_{r=1}^{n} \frac{\partial \tilde{T}}{\partial \omega_{r}} W_{\mu}^{r}-\tilde{Q}_{\mu}+ \\
+ & \sum_{\beta=1}^{b}\left\{\frac{1}{p}\left[\frac{\partial \tilde{T}^{(p)}}{\partial \omega_{\beta}^{(p-1)}}-(p+1) \frac{\partial \tilde{T}}{\partial \pi_{\beta}}\right]+\sum_{r=1}^{n} \frac{\partial \tilde{T}}{\partial \omega_{r}} W_{\beta}^{r}-\tilde{Q}_{\beta}\right\} \frac{\partial \Omega_{\beta}^{(p-1)}}{\partial \omega_{\mu}^{(p-1)}}=0 .
\end{aligned}
$$

We refer to (28) as the generalized programmed motion equations (GPME) in quasi-coordinates. For $p=1$, equations (28) become (15). They may be presented in a form similar to (18)

$$
\begin{gathered}
M(q) \dot{\omega}+C(q, \omega)+D(q)=\tilde{Q}, \\
\widetilde{G}_{\beta}\left(t, q_{\sigma}, \omega_{r}, \dot{\omega}_{r}, \ldots, \omega_{r}^{(p-1)}\right)=0
\end{gathered}
$$

\section{Design of a control strategy based on the GPME in quasi-coordinates}

We have reported the derivation of the generalized programmed motion equations (GPME) in quasicoordinates. They enable deriving a constrained system dynamics with $\omega_{\beta}^{(p-1)}=\Omega_{\beta}^{(p-1)}\left(t, q_{\sigma}, \omega_{\sigma}, \omega_{\sigma}, \ldots, \omega_{\mu}^{(p-1)}\right)$. If the constraints specify a task to be done or motion to be followed, a question arises - how to execute this task and how to track the desired motion?

A control strategy dedicated to track predefined motions is referred to as the model reference tracking control strategy for programmed motion. It is based on two dynamic models derived in quasi-coordinates:

1. The reference dynamic model. It governs motion equations of a system subjected to $\mathrm{NC}$, either material, programmed or both. This is the reference dynamics block of the form (29).

2. The dynamic control model. It takes into account only material constraints and conservation laws on the system. This is the control dynamics block (18).

Outputs of the reference dynamics are inputs to the control law and the control dynamics.

Architecture of the tracking strategy is designed in such a way that it separates the non-material and material constraints. They are merged into separate models. It gives rise to an idea of a derivation of both dynamic models using other set of coordinates.

The reference dynamics (29) serves programmed motion planning in the sense that solutions of (29) deliver programmed motions patterns, which may be verified if a system for which the program is specified can perform it. The control goal is as follows: Given a programmed motion specified by the constraints (22) and the system reference dynamics (29), design a feedback controller to track the desired programmed motion.

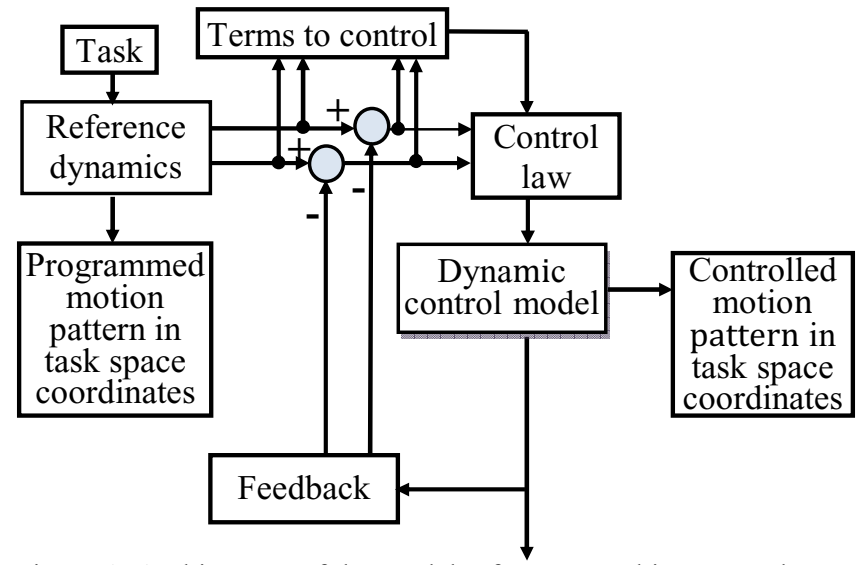

Figure 1. Architecture of the model reference tracking control strategy for programmed motion. 
The strategy for programmed motion tracking is not sensitive to the constraint order and type, and the NS design. This is in contrast to many control designs, in which each constraint type is treated separately and a controller is modified for each of them.

\section{Examples}

\subsection{Example 1 - motion control of a car with a trailer}

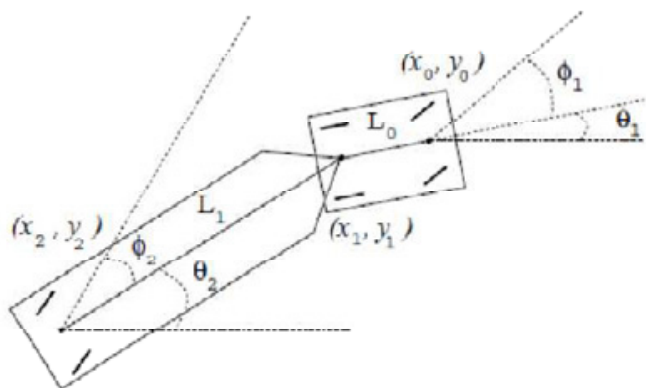

Figure 2. A car with a trailer model.

A car with a trailer model presented in fig. 2 consists of three pair of wheels, which are replaced by unicycles. According to the figure, the coordinate vector is $q=\left(x_{1}, y_{1}, \theta_{1}, \Phi_{1}, \theta_{2}, \Phi_{2}\right)$. The wheels do not slip and the three Nonholonomic equations have the form

$$
\begin{gathered}
\dot{x}_{1} \sin \left(\theta_{1}+\Phi_{1}\right)-\dot{y}_{1} \cos \left(\theta_{1}+\Phi_{1}\right)-\dot{\theta}_{1} L_{0} \cos \Phi_{1}=0 \\
\dot{x}_{1} \sin \theta_{1}-\dot{y}_{1} \cos \theta_{1}=0 \\
\dot{x}_{1} \sin \left(\theta_{2}+\Phi_{2}\right)-\dot{y}_{1} \cos \left(\theta_{2}+\Phi_{2}\right)+\dot{\theta}_{2} L_{1} \cos \Phi_{2}=0
\end{gathered}
$$

The quasi-velocities are introduced such that they naturally conform to the car driving, i.e.

$$
\begin{gathered}
\omega_{1}=V=\dot{x}_{1} \cos \left(\theta_{1}\right)+\dot{y}_{1} \sin \left(\theta_{1}\right) \\
\omega_{2}=\dot{\phi}_{1} \\
\omega_{3}=\dot{\phi}_{2} \\
\omega_{4}=\dot{x}_{1} \sin \left(\theta_{1}+\Phi_{1}\right)-\dot{y}_{1} \cos \left(\theta_{1}+\Phi_{1}\right)-\dot{\theta}_{1} L_{0} \cos \Phi_{1}=0 \\
\omega_{5}=\dot{x}_{1} \sin \theta_{1}-\dot{y}_{1} \cos \theta_{1}=0 \\
\omega_{6}=\dot{x}_{1} \sin \left(\theta_{2}+\Phi_{2}\right)-\dot{y}_{1} \cos \left(\theta_{2}+\Phi_{2}\right)+\dot{\theta}_{2} L_{1} \cos \Phi_{2}=0
\end{gathered}
$$

Matlab symbolic toolbox was used to derive the Boltzmann-Hamel equations and its control dynamics form. Due to the complexity of the equations, their final form is (after canceling $\omega_{4}, \omega_{5}$ and $\omega_{6}$.)

$$
M(q) \dot{\omega}+C(q, \omega)=\tau
$$

with

$$
M=\left[\begin{array}{ccc}
M_{1} & & \\
& I_{k 1} & \\
& & I_{k 2}
\end{array}\right], \quad C=\left[\begin{array}{c}
M_{2} \omega_{1} \omega_{3}+M_{3} \omega_{1}^{2}+M_{4} \omega_{1} \omega_{2} \\
0 \\
0
\end{array}\right]
$$

and $\omega=\left(\omega_{1}, \omega_{2}, \omega_{3}\right)$

The control goal is to drive along a circle so the programmed constraint is a desired trajectory for $\left(x_{1}, y_{l}\right)$. It is presented in fig. 3 .

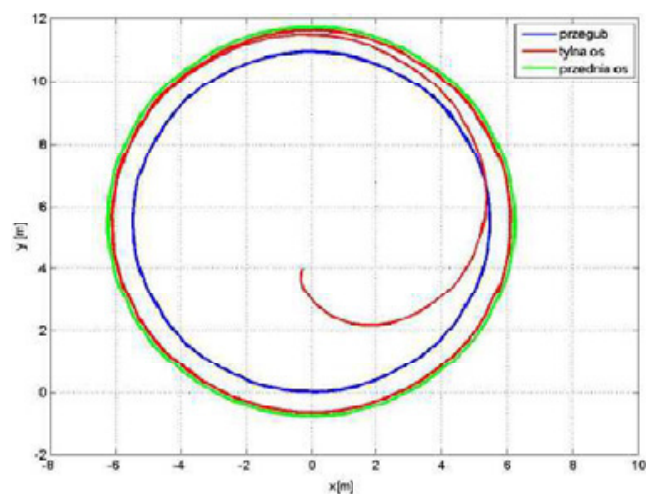

Figure 3. Driving a prescribed trajectory by a car with a trailer.

\subsection{Example 2 - motion control of an underactuated 2-link planar manipulator}

A 2-link planar manipulator is a holonomic system. We make it nonholonomic by an imposition of the $\mathrm{NC}$ on it and underactuated by removing the second actuator.

It moves in the horizontal plane $(x, y)$. Two degrees of freedom are described by $\Theta_{1}, \Theta_{2}$.

We formulate a programmed constraint that the manipulator end-effector is to move along a trajectory for which its curvature changes according to a specified function $\Phi^{*}=\frac{d \Phi(t)}{d t}$. It has the form

$$
\dddot{x}=\frac{-\Phi\left(\dot{x}^{2}+\dot{y}^{2}\right)^{2}\left[\dot{\Phi}\left(\dot{x}^{2}+\dot{y}^{2}\right)+3 \Phi(\ddot{x} \ddot{x}+\ddot{y} \ddot{y})\right]}{\dot{y}(\ddot{x} \ddot{y}-\dddot{x} \ddot{y})}+\dddot{y} \frac{\dot{x}}{\dot{y}} .
$$

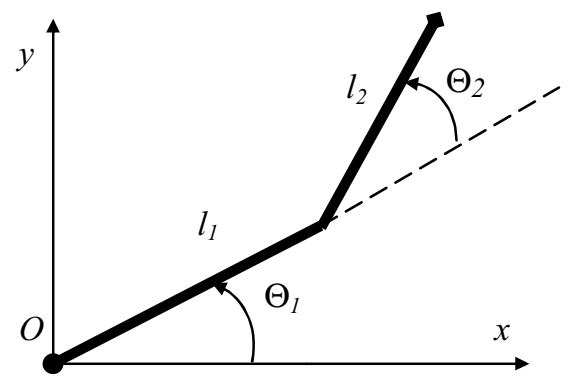

Figure 4. Two-link planar manipulator model.

Quasi-coordinates may be selected as

$$
\omega_{1}=\dot{\Theta}_{1} l_{1}, \quad \omega_{2}=\left(\dot{\Theta}_{1}+\dot{\Theta}_{2}\right) l_{2} .
$$

The programmed constraint in quasi-velocities is

$$
\ddot{\omega}_{2}-\left(1-F_{2}\right) \frac{l_{2}}{l_{1}} \ddot{\omega}_{1}-F_{1} l_{2}=0,
$$

where $F_{1}$ and $F_{2}$ are functions of the manipulator geometric and inertia properties, $\Phi, \omega_{1}, \omega_{2}$ and their first order time derivatives.

The reference dynamics (29) has the form

$$
\begin{gathered}
{\left[b_{1}-b_{2}-F_{2}\left(b_{2}-\delta\right)\right] l_{2} \dot{\omega}_{1}+\left[b_{2}-F_{2} \delta\right] \dot{\omega}_{2} l_{1}+c l_{1} l_{2}=0,} \\
\ddot{\omega}_{2} l_{1}-\left(1-F_{2}\right) l_{2} \ddot{\omega}_{1}-F_{1} l_{2} l_{1}=0 . \\
\omega_{1}=\dot{\Theta}_{1} l_{1}, \\
\omega_{2}=\left(\dot{\Theta}_{1}+\dot{\Theta}_{2}\right) l_{2} .
\end{gathered}
$$

The control dynamics (18) become 


$$
\begin{aligned}
& \dot{\omega}_{1}=u_{1}, \\
& \dot{\omega}_{2}=\frac{-\beta l_{2} \cos \Theta_{2}}{\delta l_{1}} \dot{\omega}_{1}-\frac{-\beta l_{2} \sin \Theta_{2}}{\delta l_{1}^{2}} \omega_{1}^{2} . \\
& \omega_{1}=\dot{\Theta}_{1} l_{1}, \\
& \omega_{2}=\left(\dot{\Theta}_{1}+\dot{\Theta}_{2}\right) l_{2} .
\end{aligned}
$$

Tracking the programmed motion in the $(x, y)$ plane using the PD controller is presented in fig.5.

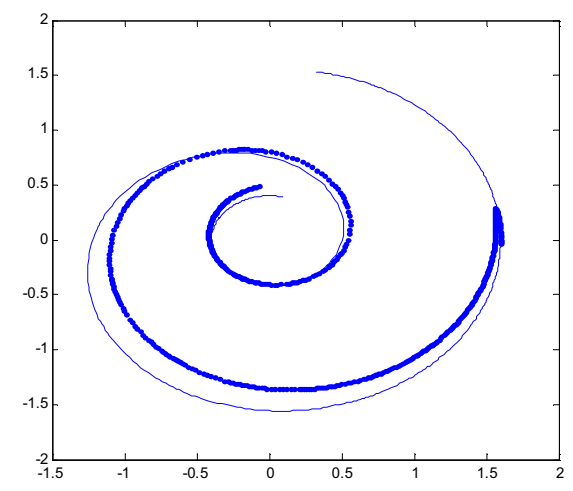

Figure 5. Tracking by the PD controller.

Modeling and the controller design for the manipulator model in quasi-coordinates result in the compact forms of the reference and control dynamics. Simulations are faster and numerical stabilization of the constraint equations is not needed.

\section{Conclusions}

In the paper we develop the theoretic model-based control oriented modeling framework. It yields equations of motion for a NS in quasi-coordinates. We demonstrate that the framework may offer a fast way to obtain equations of motion for a constrained system either for the dynamic analysis or control. The theoretic modelbased control oriented modeling framework treats the two types of constraints in the same way in modelling and a controller design. Simulation results confirm that modelbased control oriented modeling in quasi-coordinates is efficient and it supports numerical stabilization of the NC equations. Future research is planned in the area of design controllers using quasi-velocities description to fully exploit properties of motion equations in quasicoordinates and quasi-velocities.

The research was done under grant 2011/01/B/ST10/06966 from Polish National Science Centre.

\section{References}

1. J.M. Cameron and W.J. Book, Modeling mechanisms with nonholonomic joints using the Boltzmann-Hamel equations, Int. J.Robot. Res. 16(1):47-59 (1997)

2. J.G. Papastavridis, On the Boltzmann-Hamel equations of motion: a vectorial treatment, J. Appl. Mech. 61:453-459 (1994)

3. E. Jarzębowska, Quasi-coordinates based dynamics modeling and control design for nonholonomic systems, Nonlin. Anal. 16(16):1741-1754, (2008)
4. E. Jarzębowska, Model-based tracking control of nonlinear systems (CRC Press, Boca Raton 2012)

5. E. Jarzębowska, Advanced Programmed Motion Tracking Control of Nonholonomic Mechanical Systems, IEEE Trans. Robot.,24(6):1315-1328 (2008)

6. F. Lewis, D.M. Dowson and Ch.T. Abdallach, Robot manipulator control, theory and practice (Marcel Dekker Inc., New York 2004)

7. H.G. Kwatny and G.L. Blankenship, Nonlinear control and analytical mechanics. A computational approach, (Boston: Birkhauser, 2000)

8. A.M. Bloch, Nonholonomic mechanics and control, 24 Interdisciplinary applied mathematics (Springer 2003)

9. L.S. Crawford and S.S. Sastry, Biological motor control approaches for a planar diver. Proc. Conf. Decision Control, 3881-3886 (1995)

10. K.C. Koh and H.S. Cho, A smooth path tracking algorithm for wheeled mobile robots with dynamic constraints, J. Intell. Robot. Syst., 24:367-385 (1999)

11. A. Scheuer and Ch. Laugier, Planning sub-optimal and continuous-curvature paths for car-like robots, Proc. IEEE/RSJ Int. Conf. Intell. Robot. Syst., 25-31 (1998)

12.Z. Vafa, Space manipulator motion with no satellite attitude disturbances, Proc. IEEE Int. Conf. Robot. Automat., 1770-1775 (1991)

13. G. Grioli, Particular solutions in stereodynamics, Centro Intern. Matem. Estivo, Roma, 1-65 (1972) (in Italian)

14. P. Appell, Exemple de mouvement d'un point assujeti a une liason exprimee par une relation Non lineaire entre les composantes de la vitesse, Comptes Renduss, 48-50 (1911)

15. H. Beghuin, Course de mecanique (Paris 1947)

16. R. Seifried, Dynamics of underactuated multibody systems: Modeling, control and optimal design (Solid mechanics and its applications), (Springer, New York 2013)

17. S. Macfarlane and E. Croft, Manipulator trajectory planning: design for real-time applications, IEEE Trans. Robot. Automat., 19, 1:42-51 (2003)

18. W. Chee, M. Tomizuka, S. Patwardhan et. al, Experimental study of lane change maneuver for AHS applications, Proc. Am. Control Conf., 1:139-143 (1995)

19. G. Oriolo, A. De Luca and M. Vendittelli, WMR control via dynamic feedback linearization: Design, implementation, and experimental validation, IEEE Trans. Contr. Systems Techn., 10(6):835-852 (2002)

20. Yu.K. Zotov and A.V. Tomofeyev, Controllability and stabilization of programmed motions of reversible mechanical and electromechanical systems, J. Appl. Math. Mech. 56(6):873-880 (1992)

21. Yu.K. Zotov, Controllability and stabilization of programmed motions of an automobile-type transport robot, J. Appl. Maths. Mech. 67(3):303-327 (2003)

22. J.I. Nejmark and N.A. Fufaev, Dynamics of nonholonomic systems. Am. Math. Soc. (Providence, Rhode Island 1972) 